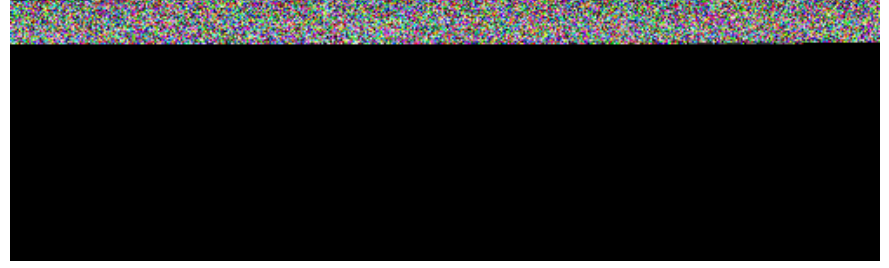

This information is current as of April 26, 2023.

Reduced Cerebral Arterial Spin-Labeled Perfusion in Children with Neurofibromatosis Type 1

K.W. Yeom, R.M. Lober, P.D. Barnes and C.J. Campen

AJNR Am J Neuroradiol 2013, 34 (9) 1823-1828

doi: https://doi.org/10.3174/ajnr.A3649

http://www.ajnr.org/content/34/9/1823 


\title{
Reduced Cerebral Arterial Spin-Labeled Perfusion in Children with Neurofibromatosis Type 1
}

\author{
K.W. Yeom, R.M. Lober, P.D. Barnes, and C.J. Campen
}

\begin{abstract}
BACKGROUND AND PURPOSE: Neurofibromatosis type 1 is associated with increased risk for stroke, cerebral vasculopathy, and neurocognitive deficits, but underlying hemodynamic changes in asymptomatic children remain poorly understood. We hypothesized that children with neurofibromatosis type 1 have decreased cerebral blood flow.
\end{abstract}

MATERIALS AND METHODS: Arterial spin-labeled CBF was measured in 14 children with neurofibromatosis type 1 (median age, 9.7 years; mean, 10.2 years; range, 22 months to 18 years) and compared with age-matched control subjects on 3T MR imaging. Three-dimensional pseudocontinuous spin-echo arterial spin-labeled technique was used. Measurements were obtained at cortical gray matter of bilateral cerebral hemispheres and centrum semiovale by use of the ROI method. Comparison by Mann-Whitney test was used, with Bonferroniadjusted $P$ values $\leq .004$ judged as significant.

RESULTS: We identified 7 of 12 areas with significantly diminished arterial spin-labeled CBF in patients with neurofibromatosis type 1 compared with control subjects. These areas included the anterior cingulate gyrus $(P=.001)$, medial frontal cortex $(P=.004)$, centrum semiovale $(P=.004)$, temporo-occipital cortex $(P=.002)$, thalamus $(P=.001)$, posterior cingulate gyrus $(P=.002)$, and occipital cortex $(P=$ $.001)$. Among patients with neurofibromatosis type 1, there were no significant differences in these regions on the basis of the presence of neurofibromatosis type 1 spots or neurocognitive deficits.

CONCLUSIONS: Reduced cerebral perfusion was seen in children with neurofibromatosis type 1, particularly in the posterior circulation and the vascular borderzones of the middle and posterior cerebral arteries.

ABBREVIATIONS: $\mathrm{ASL}=$ arterial spin-labeled; $\mathrm{NF}-1=$ neurofibromatosis type $1 ; \mathrm{UBO}=$ unidentified bright object

$\mathbf{N}$ eurofibromatosis type 1 (NF-1) is a common autosomal dominant genetic disorder affecting 1 in 3500 live births ${ }^{1}$ and frequently associated with café au lait spots, freckling, neurofibromas, optic pathway gliomas, Lisch nodules, and skeletal abnormalities. ${ }^{2}$ Other significant complications include strokes and neurocognitive dysfunction.

Risk for stroke in NF-1 has been attributed to cerebral vasculopathy, including Moyamoya syndrome., ${ }^{3,4}$ Typical vessels af-

Received October 24, 2012; accepted after revision December 9.

From the Departments of Radiology (K.W.Y., P.D.B.), Neurosurgery (R.M.L.), and Neurology (C.J.C.), Lucile Packard Children's Hospital, Stanford University, Palo Alto, California.

Abstract previously presented at: Annual Meeting of the Society for Pediatric Radiology April 2012; San Francisco, California.

Please address correspondence to Kristen W. Yeom, MD, Lucile Packard Children's Hospital, Stanford University, Department of Radiology, Pediatric MRI and CT, Room 0511, 725 Welch Rd, Palo Alto, CA 94304; e-mail: kyeom@stanford.edu

Indicates article with supplemental on-line table

Evidence-Based Medicine Level 2.

http://dx.doi.org/10.3174/ajnr.A3649 fected are, in order of occurrence, the renal, aortic, mesenteric, and cerebral arteries. ${ }^{5-7}$ There are few cerebrovascular data on the pediatric NF-1 population, but the largest series of 8 children reported vascular ectasia, stenosis, aneurysms, and Moyamoya changes on the basis of cerebral MR angiogram findings. ${ }^{3}$

Cognitive complications are relatively common in NF-1, ranging from $30-65 \%$, and include developmental delay, learning disabilities, attention deficit disorder, and headaches. ${ }^{8-11}$ Some authors have postulated that impaired cognition may arise from unidentified bright objects (UBOs), ${ }^{12-14}$ though no reliable link exists between the number of UBOs and cognitive performance. ${ }^{15-17}$ Others have suggested abnormal cortical connectivity $^{9,10}$ and underlying cerebral dysgenesis ${ }^{18}$ as sources of poor cognitive performance, whereas the relationship between NF-1 arteriopathy and cognitive impairment remains unknown.

Given the lack of cerebral hemodynamic data in the setting of risks for cerebral vasculopathy and neurocognitive complications, we sought to understand cerebral perfusion patterns in children with NF-1. Because abnormal perfusion is expected in 
patients with strokes or Moyamoya syndrome, we specifically targeted patients with NF-1 without these features. Although various MR perfusion techniques exist, we applied arterial spin-labeled (ASL) perfusion, given the unique advantages of this technique in children, including labeling efficiency, high signal-to-noise ratio, lack of contrast requirement, and its ability to directly quantify CBF. ${ }^{19,20}$ We hypothesized that children with NF-1 have decreased $\mathrm{CBF}$ as compared with normal control subjects.

\section{MATERIALS AND METHODS \\ Subjects}

All patients presenting with history of NF-1 at our children's hospital between April 2010 and April 2012 were retrospectively reviewed after approval was given by the institutional review board (IRB protocol 4223; No. 4947). The study cohort was identified on the basis of the following inclusion criteria: the patient obtained ASL perfusion at $3 \mathrm{~T}$ as part of routine surveillance MR imaging for NF-1. Patients presenting with acute neurologic symptoms, known underlying vasculopathy (aneurysms, arteriovenous malformations, fistulas, or steno-occlusive disease, including Moyamoya changes), prior strokes or stroke-like symptoms, radiation, or chemotherapy were excluded from the study. Patients with a history of seizures, hydrocephalus or shunt placement, underlying cardiac diseases, or abnormal blood pressures, including hypertension from underlying NF-1-related renal artery stenosis, were also excluded, given their potential effects on CBF. Chart review of these patients was also performed to document the presence of neurocognitive deficits. Patients were considered to have neurocognitive deficits if chart review indicated developmental delay, learning disability, or attention deficit disorder or if they required special education, for example, an individualized education plan on the basis of clinical history and academic performance. Documentation was considered complete if the chart review specifically mentioned that no such deficits were present.

\section{Control Subjects}

Age-matched control subjects who had obtained ASL perfusion at $3 \mathrm{~T}$ as part of routine brain MR imaging were recruited from our data base. The control group consisted of patients with no underlying neurologic disease and normal brain MR imaging. Examples of clinical reasons for imaging in the control group included isolated headaches, dizziness, family history of aneurysms, cholesteatoma of the middle ear, isolated facial lesions (eg, hemangioma), or ophthalmologic work-up (strabismus) without correlative orbital/brain pathology or neurologic symptoms. Children with other neurocutaneous syndromes, psychiatric diseases, migraines, and any chronic diseases, including seizures, cancer, as well as previously treated neurologic diseases, and prior or ongoing medical therapy were excluded from the control group.

\section{Imaging Methods}

All patients were examined with brain MR imaging at 3T (Discovery 750; GE Healthcare, Milwaukee, Wisconsin), by use of an 8-channel head coil. The technique used to perform perfusion ASL MR imaging has been described in detail elsewhere. ${ }^{21}$ Briefly, our vendor-supplied ASL was performed by use of a pseudocontinuous labeling period of $1500 \mathrm{~ms}$, followed by a 1500 -ms postlabel delay. Whole-brain images were acquired with a 3D back-

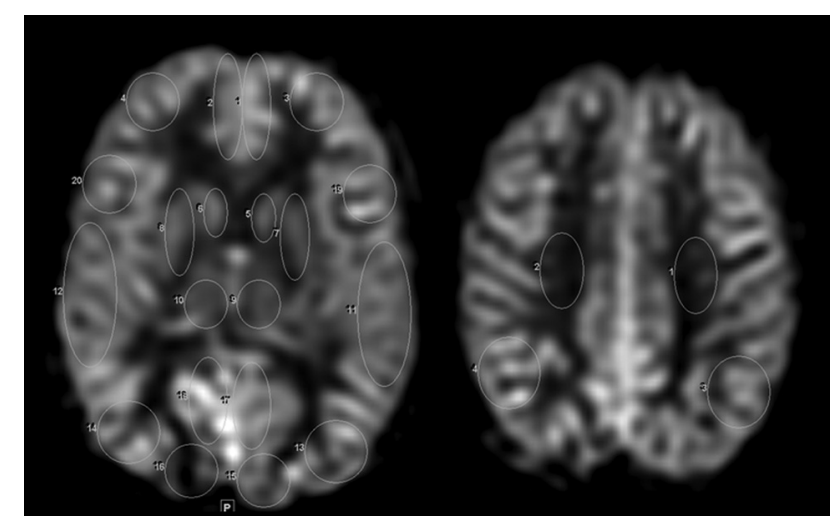

FIG 1. Brain ROI placement. ROI placement is shown in the cortical regions of the bilateral cerebral hemispheres, the deep gray nuclei, and in the centrum semiovale at 2 axial levels: at the level of the thalamus and centrum semiovale.

ground-suppressed FSE stack-of-spirals method, with a TR of approximately 5 seconds. Multi-arm spiral imaging was used, with 8 arms and 512 points acquired on each arm (bandwidth, $62.5 \mathrm{kHz}$ ), yielding in-plane and through-plane spatial resolution of 3 and $4 \mathrm{~mm}$, respectively. A high level of background suppression was achieved by the use of 4 separate inversion pulses spaced around the pseudocontinuous labeling pulse. The sequence required 5 minutes to acquire, which included proton attenuation images required for CBF quantification. For graphic prescription of the ASL, the sagittal image following the 3-plane localizer was used for alignment. Postprocessing was performed by use of an automated reconstruction script that returned CBF images directly to the scanner console within 1 minute, by use of the microsphere methodology described by Buxton et al. ${ }^{22}$ Other ASL MR imaging parameters were TR/TE, 4632/10.5; FOV, $24 \times 24$ $\mathrm{cm}$; matrix, $512 \times 8$; and NEX of 3 . Conventional MR imaging was also performed, including T1 FLAIR, T2 FSE, FLAIR, T1 spoiled gradient-recalled, DWI/DTI, gradient-recalled, and 3-plane T1 spoiled gradient-recalled and T1 spin-echo gadolinium-enhanced sequences.

\section{Imaging Analysis}

Quantitative cerebral cortical CBF was measured at 2 axial sections at the level of the thalamus and the centrum semiovale. Fourteen cortical ROIs and 4 ROIs of the deep gray nuclei were obtained at the level of the thalamus; an additional 4 ROIs were obtained at the level of the centrum semiovale, as shown in Fig 1. These regions included anterior and posterior cingulate gyri, medial frontal lobe, frontal operculum, anterior caudate nucleus, globus pallidus, thalamus, superiomedial temporal cortex, temporo-occipital cortex, occipital cortex, centrum semiovale, and parietal cortex. The ROI sizes ranged from $100-800 \mathrm{~mm}^{2}$, depending on the size of the structure interrogated. A board-certified neuroradiologist (K.W.Y.) with added Certificate of Added Qualification (7 years of experience) selected ROIs, blinded to clinical information and at the same anatomic levels in all patients. A second blinded board-certified neuroradiologist (P.D.B.) with a Certificate of Added Qualification ( $>30$ years of experience) independently confirmed appropriate anatomic placement of the ROI. To test for significant differences in CBF 
Table 1: Patient characteristics and clinical data $(n=14)$

\begin{tabular}{lc}
\hline Age of diagnosis, $y$ & \\
Median & 9.7 \\
Range & $1.8-18$ \\
Sex & \\
$\quad$ Male & $7(50 \%)$ \\
Female & $7(50 \%)$ \\
MRI findings & \\
$\quad$ NF-1 spots & $14(100 \%)$ \\
Optic nerve/chiasm thickening & $7(50 \%)$ \\
Plexiform neurofibroma & $6(43 \%)$ \\
Clinical findings & \\
Headaches & $6(43 \%)$ \\
Cognitive or speech delay & $5(36 \%)$ \\
ADHD & $2(14 \%)$ \\
\hline
\end{tabular}

Note:-ADHD indicates attention deficit/hyperactivity disorder.

between the patients and control subjects, comparison by MannWhitney test was used, with Bonferroni-adjusted $P$ value $\leq .004$ judged as significant. To test for difference in cerebral perfusion in the brain regions between the sedated and nonsedated groups, as well as between boys and girls, independent samples of the MannWhitney test were used, with Bonferroni-corrected $\alpha=0.004$ (for 12 comparisons).

\section{RESULTS}

\section{Clinical Findings}

Fourteen children met clinical criteria for NF-1 (median age, 9.7 years; mean, 10.2 years; range, 22 months to 18 years) and were included in the study. There were 7 boys and 7 girls. Patient demographics and clinical data are shown in Table 1.

Four patients were completely asymptomatic except for cutaneous manifestations of NF-1 (eg, café au lait spots, axillary freckling, Lisch nodules, and plexiform neurofibromata) without gross neurocognitive, neuro-opthalmologic, or behavioral disorders.

Six patients reported chronic or occasional headaches. Five children had cognitive, speech, or intellectual difficulties requiring special education or various forms of neurocognitive therapy. Two children had attention deficit disorder and difficulty focusing at school but were not receiving medical therapy at the time of the study.

Six patients had optic nerve or chiasm thickening (4 showed no enhancement; 2 showed mild enhancement) that was conservatively managed at the time of the study. In 1 patient, T2-highintensity abnormality was present in left optic tract and portion of the optic radiation without associated abnormal enhancement or signal extending into the posterior regions of the optic radiation or the occipital lobe. Of the 6 patients with thickening or enhancement of the optic apparatus, 4 had no visual acuity or field deficits, 2 required corrective lenses for decreased visual acuity (20/30 and 20/20; 20/400 and 20/30), and 1 patient (22 months old) demonstrated gross asymmetry in visual acuity (by assessing the child's reaction to covering one of the eyes) but without a field cut. All 6 patients with optic nerve or chiasm thickening were deemed stable by ophthalmologic examination and required no therapy at the time of this study.

All patients demonstrated multiple NF-1 spots on their surveillance T2-weighted MR imaging scans predominantly in the basal ganglia, thalami, brain stem, and cerebellum. In the 4 older children (ages 13-18 years), these NF-1 spots were relatively faint in appearance. Except for characteristic NF-1 spots and optic

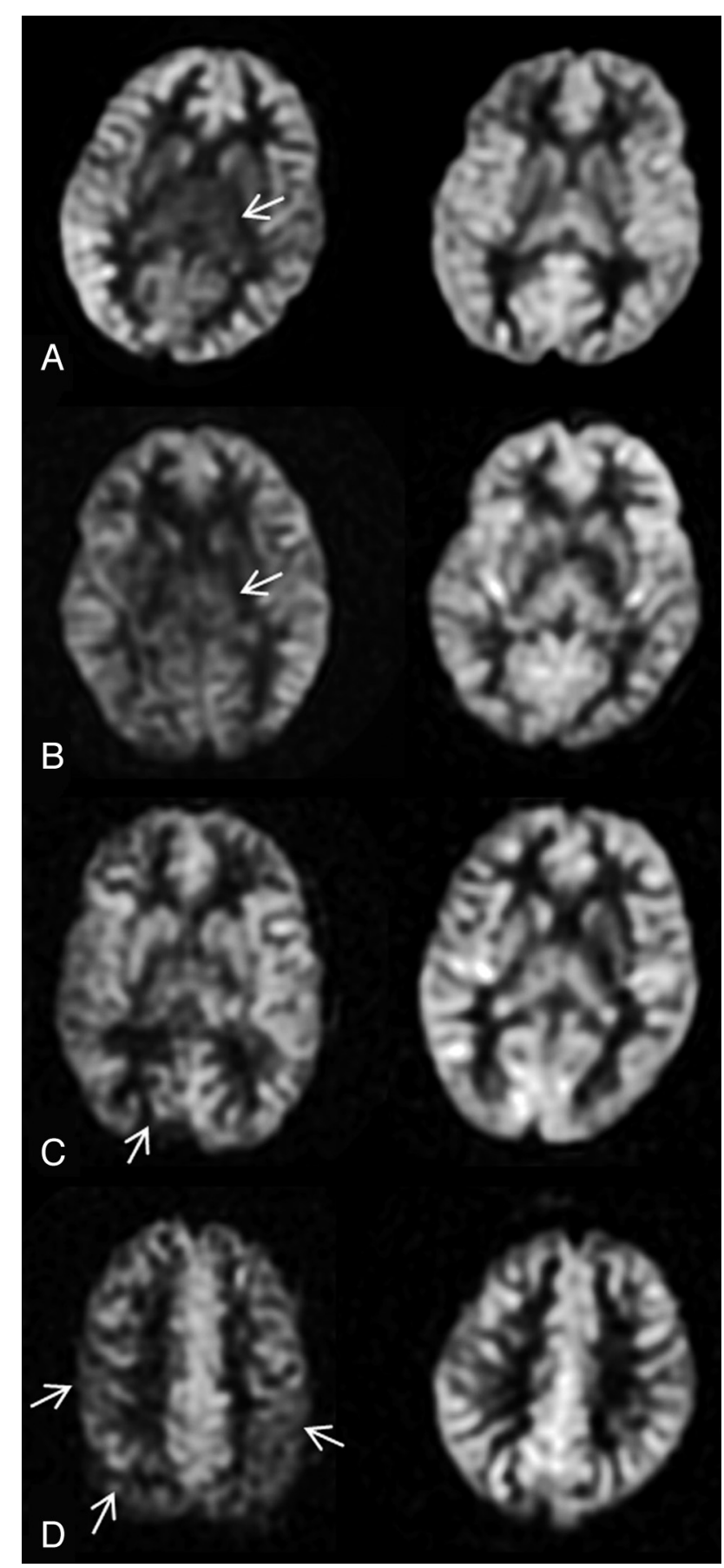

FIG 2. Various patterns of reduced cerebral perfusion in patients with NF-1 are shown compared with age-matched control subjects. Note marked hypoperfusion of bilateral thalami (arrows) in a 7-year-old girl $(A)$ and a 13-year-old boy $(B)$ with NF-1 in the setting of global and patchy hypoperfusion. More focal reduced perfusion is seen in the right occipital cortex (arrow) superimposed on heterogeneous hypoperfusion in a 10-year-old boy with NF-1 (C). More superiorly, there are additional patchy areas of hypoperfusion in this child $(D)$ with asymmetric low ASL signal in bilateral frontoparietal regions (arrows).

nerve/chiasm thickening, no other abnormality, including mass, abnormal brain enhancement, or dysgenesis/dysplasia, was present on conventional MR imaging.

\section{Cerebral Blood Flow}

Reduced CBF was seen in children with NF-1 compared with control subjects (Fig 2). The most significant difference in perfu- 
Table 2: Comparison of CBF in patients with NF-1 and control subjects by anatomic location and vascular territories

\begin{tabular}{|c|c|c|c|}
\hline Structure & $\begin{array}{c}\text { Control CBF, } \\
\mathrm{mL} / 100 \mathrm{mg} \\
\text { per minute }(n=14)\end{array}$ & $\begin{array}{c}\text { NF-1 CBF, } \\
\mathrm{mL} / 100 \mathrm{mg} \\
\text { per minute }(n=14)\end{array}$ & $\begin{array}{c}\text { Mann-Whitney } \\
P \text { Value } \\
\end{array}$ \\
\hline \multicolumn{4}{|l|}{ ACA territory } \\
\hline Anterior cingulate gyrus & $71 \pm 7$ & $61 \pm 8$ & ${ }^{\mathrm{a}} .001$ \\
\hline Medial frontal cortex & $65 \pm 7$ & $56 \pm 9$ & ${ }^{\mathrm{a}} .004$ \\
\hline Anterior caudate nucleus & $57 \pm 9$ & $51 \pm 4$ & .013 \\
\hline \multicolumn{4}{|l|}{ ACA-MCA borderzone } \\
\hline Centrum semiovale & $28 \pm 5$ & $22 \pm 4$ & ${ }^{\mathrm{a}} .004$ \\
\hline \multicolumn{4}{|l|}{ MCA territory } \\
\hline Frontal operculum & $68 \pm 8$ & $60 \pm 8$ & .013 \\
\hline Globus pallidus & $50 \pm 6$ & $44 \pm 6$ & .012 \\
\hline \multicolumn{4}{|l|}{ MCA-PCA borderzone } \\
\hline Parietal cortex & $65 \pm 9$ & $55 \pm 9$ & .008 \\
\hline Superomedial temporal cortex & $67 \pm 9$ & $60 \pm 8$ & .012 \\
\hline Temporo-occipital cortex & $64 \pm 10$ & $51 \pm 8$ & ${ }^{\mathrm{a}} .002$ \\
\hline \multicolumn{4}{|l|}{ Anterior choroidal-PcomA-PCA } \\
\hline Thalamus & $55 \pm 10$ & $44 \pm 8$ & ${ }^{\mathrm{a}} .001$ \\
\hline \multicolumn{4}{|l|}{ PCA territory } \\
\hline Posterior cingulate gyrus & $70 \pm 13$ & $57 \pm 8$ & ${ }^{\mathrm{a}} .002$ \\
\hline Occipital cortex & $61 \pm 13$ & $45 \pm 10$ & ${ }^{\mathrm{a}} .001$ \\
\hline
\end{tabular}

Note:-ACA indicates anterior cerebral artery; PCA, posterior cerebral artery; PcomA, posterior communicating artery. ${ }^{a}$ Significant at $\leq .004$

sion was seen in the anterior cingulate gyrus $(P=.001)$, medial frontal lobe $(P=.004)$, centrum semiovale $(P=.004)$, temporooccipital lobe $(P=.002)$, thalamus $(P=.001)$, posterior cingulate gyrus $(P=.002)$, and the occipital lobe $(P=.001)$. The quantitative results of the CBF values by specific brain regions and vascular territories are summarized in Table 2.

No NF-1 spots were present within the ROIs of the cortical regions and the centrum semiovale; varying degrees of NF-1 spots were present within the ROIs of the deep gray nuclei. There were no significant differences in these regions on the basis of the presence or number of NF-1 spots, optic pathway glioma, or neurocognitive deficits (On-line Table).

Of 28 children (14 NF-1 and 14 control subjects), 12 received sedation and 16 did not require sedation. The mean ages of those patients were $7.1 \pm 3.0$ years and $13.4 \pm 4.4$ years, respectively (independent samples Mann-Whitney $U$ test $P<.001$ ). There was no difference in cerebral perfusion at any location between these groups (independent-samples Mann-Whitney $U$ test; Bonferroni-corrected $\alpha=0.004$ for 12 comparisons). There were 17 boys and 11 girls in our cohort; no difference in cerebral perfusion at any location between these 2 groups was seen (independentsamples Mann-Whitney $U$ test; Bonferroni-corrected $\alpha=0.004$ for 12 comparisons).

\section{DISCUSSION}

These results demonstrate significantly lower CBF in patients with NF-1 compared with control subjects, occurring most prominently in the posterior circulation and the borderzones of the middle and posterior cerebral arteries. To our knowledge, this is the first study to report perfusion abnormalities in children with NF-1 in the absence of prior strokes or underlying Moyamoya syndrome. There are 2 potential mechanisms to account for this: 1 ) vasculopathy and associated steno-occlusive changes in the cerebral microvasculature and 2) alteration in cerebral metabolic demand.

Children with NF-1 are known to harbor cerebral vasculopathy. ${ }^{3}$ Vascular proliferative and dysplastic changes such as con- centric intimal thickening, spindle cell proliferation, and disruption of the internal elastic have also been observed in brain specimens of patients with NF-1. ${ }^{23}$ Reduced expression of neurofibromin, the NF-1 gene product important for maintenance of the endothelial layer, may disrupt vascular wall integrity ${ }^{7}$ and result in intimal thickening and arterial stenosis through the Ras signaling system and smooth muscle proliferation. ${ }^{24}$

The reasons for more dominant perfusion deficits in the posterior and border zones remain unclear. Vasoconstriction or hypoperfusion superimposed on vaso-autoregulatory dysfunction at these sites ${ }^{25-27}$ may contribute to this pattern, similar to posterior reversible encephalopathy syndrome. ${ }^{28}$ It is also possible that there are variations of neurofibromin expression within the different regions of the cerebral vasculature, though this remains unknown.

Alternatively, decreased CBF could reflect underlying reduced cerebral metabolic requirement in children with NF-1. A potential biomarker of regional brain function, $\mathrm{CBF}$ is tightly coupled to brain metabolism ${ }^{29,30}$ and may thereby provide information similar to FDG-PET, which is sensitive in detecting disease-related functional changes. ${ }^{31}$ Similar results derived from FDG-PET metabolism, ${ }^{15} \mathrm{O}$-PET perfusion, and ASL perfusion in healthy adults and those with neurodegenerative conditions ${ }^{32-34}$ suggest that neuronal glucose metabolism could be inferred from CBF techniques.

Prior FDG-PET studies in NF-1 ${ }^{35-37}$ have shown abnormal cerebral metabolism similar in distribution to the perfusion pattern noted in this study, for example, global hypometabolism with a more marked hypometabolism in the posterior brain regions. For example, in 4 adolescents with NF-1 (9-20 years of age), global hypometabolism with a more marked reduction of FDG activity in the thalamus and the occipital lobes was noted with intact metabolism in the striatum. ${ }^{35}$ Another study of 10 children with NF-1 (4-15 years of age) observed heterogeneously decreased cortical FDG uptake. Although regional cerebral metabolic differences were not quantitatively analyzed in that study, profound hypometabolism in the thalamus was observed. ${ }^{36} \mathrm{~A}$ study of 20 adults with NF-1 also showed reduced FDG-PET metabolism in the thalamus, and to a lesser degree, in the cerebellum. In these studies, the extent of abnormal cerebral metabolism did not correlate with the presence or number of UBOs, ${ }^{35,37}$ degree of cognitive impairment, ${ }^{35}$ or differences in age or sex, ${ }^{37}$ similar to the present study.

Although a primary defect of the thalamus or cerebral cortex has not been defined, investigators have postulated that dysfunction in the cortical-thalamic relationship is a potential source of neurocognitive complications in NF-1. ${ }^{17,36,37}$ Marked reduction in $\mathrm{CBF}$ in the thalamus and variable alterations in cortical perfusion in our study also implicate these brain regions, potentially 
reflecting underlying dysgenesis or a dysfunction in their relationship, or underlying arteriopathy as a source of cognitive deficits, not unlike the adult model for vascular dementia. ${ }^{38}$

In the present study, we used ASL perfusion to measure CBF because it directly quantifies CBF by use of in-flowing arterial protons as an endogenous tracer ${ }^{39,40}$ and has additional advantages in children because of the high labeling efficiency and signalto-noise ratio. ${ }^{19}$ Direct comparisons of ASL with ${ }^{15} \mathrm{O}$-PET have also shown that MR imaging and PET methods for measuring $\mathrm{CBF}$ are highly comparable ${ }^{41}$ and reproducible across variable time intervals, ${ }^{42,43}$ and therefore ASL is a reliable perfusion method that could also be clinically implemented by use of standard MR imaging equipment and without the need for ionizing radiation or contrast.

Limitations of this study included small sample size and a tertiary care center referral pattern that may attract a more severely affected NF-1 population. Therefore, our results may overestimate cerebral perfusion differences in typical patients with NF-1. Although we did not observe worse perfusion in those reported to have cognitive dysfunction, our analysis was based on the need for special education or cognitive therapy and lacked standardized psychiatric tests. As such, subtle deficits could have been overlooked, and, in the very young, cognitive changes may be difficult to identify or have yet to emerge. Of note, none of our patients underwent cervical or intracranial MRA/CTA because they were not suspected of strokes or vascular anomalies, and it is possible that occult cervical vascular anomalies could have affected cerebral perfusion. Given other causes for cerebral perfusion abnormality, such as seizures, migraines, medications, and changes in cardiac output and blood pressure status, we excluded patients with any acute symptoms at the time of imaging or chronic conditions that might alter cerebral perfusion, and therefore these factors probably did not affect the study.

\section{CONCLUSIONS}

Reduced cerebral perfusion was seen in children with NF-1, most significantly in the posterior circulation and borderzones of the middle and posterior cerebral arteries. Future studies may demonstrate an important role for ASL perfusion in the presymptomatic diagnosis of NF-1-related cerebral vasculopathy and the definition of NF-1-related vasculopathy patterns and in providing a link between cerebral perfusion and cognitive performance in NF-1.

\section{REFERENCES}

1. Friedman JM. Epidemiology of neurofibromatosis type 1. Am J Med Genet 1999;89:1-6

2. Neurofibromatosis. Conference statement. National Institutes of Health Consensus Development Conference. Arch Neurol 1998;45:575-78

3. Rosser TL, Vezina G, Packer RJ. Cerebovascular abnormalities in a population of children with neurofibromatosis type 1. Neurology 2005;64:553-55

4. Sobata E, Ohkuma H, Suzuki S. Cerebrovascular disorders associated with von Recklinghausen's neurofibromatosis: a case report. Neurosurgery 1998;22:544-49

5. Hamilton SJ, Friedman JM. Insights into the pathogenesis of neurofibromatosis 1 vasculopathy. Clin Genet 2000;58:341-44

6. Friedman JM, Arbiser J, Epstein JA, et al. Cardiovascular disease in neurofibromatosis 1: report of the NF1 Cardiovascular Task Force. Genet Med 2002;4:105-11

7. Norton KK, Xu J, Gutmann DH. Expression of the neurofibromatosis 1 gene product, neurofibromin, in blood vessel endothelial cells and smooth muscle. Neurobiol Dis 1995;2:13-21

8. North K, Hyman S, Barton B. Cognitive deficits in neurofibromatosis1. J Child Neurol 2002;17:650-12

9. North KN, Riccardi V, SamangoSprouse C, et al. Cognitive function and academic performance in neurofibromatosis 1 : consensus statement from the NF1 Cognitive Disorder Task Force. Neurology 1997;48:1121-27

10. North K. Neurofibromatosis type 1. Am JMed Genet 2000;97:119-27

11. Hyman SL, Shores A, North KN. The nature and frequency of cognitive deficits in children with neurofibromatosis type 1. Neurology 2005;65:1037-44

12. North K, Joy $\mathrm{P}$, Yuille D, et al. Specific learning disability in children with neurofibromatosis type 1: significance of MRI abnormalities. Neurology 1994;44:878-83

13. Mott SH, Skyrja PA, Baumgardner TL, et al. Neurofibromatosis type 1: association between volumes of T2-weighted signals on MRI in young children with neurofibromatosis type 1 correlating with cognitive scores, abstract. Pediatr Neurol 1994;11:88

14. Feldmann R, Schuierer G, Wessel A, et al. Development of MRI T2 hyperintensities and cognitive functioning in patients with neurofibromatosis type 1. Acta Paediatr 2010;99:1657-60

15. Duffner PK, Cohen ME, Seidel FG, et al. The significance of MRI abnormalities in children with neurofibromatosis. Neurology 1989;39:373-78

16. Ferner RE, Chaudhuri R, Bingham J, et al. MRI in neurofibromatosis 1: the nature and evolution of increased intensity T2 weighted lesions and their relationship to intellectual impairment. J Neurol Neurosurg Psychiatry 1993;56:492-95

17. Moore BD, Slopis JM, Schomer D, et al. Neuropsychological significance of areas of high signal intensity on brain magnetic resonance imaging scans of children with neurofibromatosis. Neurology 1996;46:1660-68

18. Rosman NP, Pearce J. The brain in multiple neurofibromatosis (von Recklinghausen disease): a suggested neuropathological basis for the associated mental defect. Brain 1967;90:829-38

19. Wang J, Licht DJ, Jahng GH, et al. Pediatric perfusion imaging using pulsed arterial spin labeling. J Magn Reson Imaging 2003;18:404-13

20. Pollock JM, Tan H, Kraft RA, et al. Arterial spin-labeled MR perfusion imaging: clinical applications. Magn Reson Imaging Clin North Am 2009;17:315-38

21. Dai W, Garcia D, Bazelaire C, et al. Continuous flow-driven inversion for arterial spin labeling using pulsed radio frequency and gradient fields. Magn Reson Med 2008;60:1488-97

22. Buxton RB, Frank LR, Wong EC, et al. A general kinetic model for quantitative perfusion imaging with arterial spin labeling. Magn Reson Med 1998;40:383-96

23. Kwong KL, Wong YC. Moyamoya disease in a child with neurofibromatosis type-1. J Paediatr Child Health 1999;35:108-09

24. Tan RM, Chang SM, Seow WT, et al. 'Moya' than meets the eye: neurofibromatosis type 1 associated with Moyamoya syndrome. Singapore Med J 2008;49:107-09

25. Beausang-Linder M, Bill A. Cerebral circulation in acute arterial hypertension-protective effects of sympathetic nervous activity. Acta Physiol Scand 1981;111:193-99

26. Zwienenberg-Lee M, Muizelaar JP. Clinical pathophysiology of traumatic brain injury. In: Winn HR, ed. Youman's Neurological Surgery. 5th ed. Philadelphia: Saunders; 2004:5039-64

27. Langfitt TW, Obrist WD. Occlusive cerebrovascular disease. In: Wilkins RH, Rengachary SS, ed. Neurosurgery. New York: McGrawHill; 1985:1167-73

28. Bartynski WS. Posterior reversible encephalopathy syndrome, part 2: controversies surrounding pathophysiology of vasogenic edema. AJNR Am J Neuroradiol 2009;29:1043-49

29. Raichle ME. Behind the scenes of functional brain imaging: a his- 
torical and physiological perspective. Proc Natl Acad Sci U S A 1998;95:765-72

30. Juepnert M, Wieller C. Review: does measurement of regional cerebral blood flow reflect synaptic activity? Implications for PET and fMRI. Neuroimage 1995;2:148-56

31. Landau SM, Harvey D, Madison CM, et al. Comparing predictors of conversion and decline in mild cognitive impairment. Neurology 2010;75:230-38

32. Baron JC, Lebrun-Grandie P, Collard P, et al. Noninvasive measurement of blood flow, oxygen consumption, and glucose utilization in the same brain regions in man by positron emission tomography. J Nucl Med 1982;23:391-99

33. Chen Y, Wolk DA, Reddin JS, et al. Voxel-level comparison of arterial spin-labeled perfusion MRI and FDG-PET in Alzheimer disease. Neurology 2011;77:1977-85

34. Hu WT, Wang Z, Lee VM, et al. Distinct cerebral perfusion patterns in FTLD and AD. Neurology 2010;75:881-88

35. Balestri P, Lucignani G, Fois A, et al. Cerebral glucose metabolism in neurofibromatosis 1 assessed with F-2-fluroro-2-deoxy-D-glucose and PET. J Neurol Neurosurg Psychiatry 1994;57:1479-83

36. Kaplan AM, Chen K, Lawson MA, et al. Positron emission tomography in children with neurofibromatosis 1. J Child Neurol 1997;12:499-506
37. Buchert R, von Borczyskowski D, Wilke F, et al. Reduced thalamic 18-flurodeoxyglucose retention in adults with neurofibromatosis type 1. Nucl Med Commun 2008;29:17-26

38. Baker JG, Williams AJ, Ionita CC, et al. Cerebral small vessel disease: cognition, mood, daily functioning, and imaging findings from a small pilot sample. Dement Geriatr Cogn Dis Extra 2012;2169-79

39. Alsop DC, Detre JA. Multisection cerebral blood flow MR imaging with continuous arterial spin labeling. Radiology 1998;208:410-16

40. Yang Y, Frank JA, Hou L, et al. Multislice imaging of quantitative cerebral perfusion with pulsed arterial spin labeling. Magn Reson Med 1998;39:825-32

41. Xu G, Rowley HA, Wu G, et al. Reliability and precision of pseudocontinuous arterial spin labeling perfusion MRI on $3 \mathrm{~T}$ and comparison with 15O-water PET in elderly subjects at risk for Alzheimer's disease. NMR Biomed 2010;23:286-93

42. Floyd TF, Ratcliffe SJ, Wang J, et al. Precision of the CASL-perfusion MRI technique: global and regional cerebral blood flow within vascular territories at one hour and one week. J Magn Reson Imaging 2003; 18:649-55

43. Chen Y, Wang DJ, Detre JA. Test-retest reliability of arterial spin labeling with common labeling strategies. J Magn Reson Imaging 2011;33:940-49 